\title{
Oliceridine Exhibits Improved Tolerability Compared to Morphine at Equianalgesic Conditions: Exploratory Analysis from Two Phase 3 Randomized Placebo and Active Controlled Trials
}

\author{
Gregory B. Hammer - Ashish K. Khanna - Cathy Michalsky • \\ Linda Wase · Mark A. Demitrack · Roderick Little · Michael J. Fossler • \\ Sabry Ayad
}

Received: June 4, 2021 / Accepted: July 19, 2021 / Published online: August 5, 2021

(C) The Author(s) 2021, corrected publication 2021

\section{ABSTRACT}

Introduction: In the management of postoperative acute moderate-to-severe pain, opioids remain an important component. However, conventional opioids have a narrow therapeutic index and are associated with dose-limiting

Supplementary Information The online version contains supplementary material available at https:// doi.org/10.1007/s40122-021-00299-0.

G. B. Hammer ( $₫)$

Department of Anesthesiology, Perioperative and Pain Medicine, Stanford University School of Medicine, Stanford, CA 94305-5640, USA

e-mail: ham@stanford.edu

\section{A. K. Khanna}

Department of Anesthesiology, Section on Critical Care Medicine, Wake Forest School of Medicine, Winston-Salem, NC, USA

C. Michalsky · L. Wase · M. A. Demitrack .

M. J. Fossler

Trevena Inc., Chesterbrook, PA, USA

R. Little

Department of Statistics, School of Public Health, University of Michigan, Ann Arbor, MI, USA

S. Ayad

Department of Outcomes Research, Cleveland Clinic's Anesthesiology Institute, Fairview Hospital, Cleveland, $\mathrm{OH}$, USA

A. K. Khanna · S. Ayad

Outcomes Research Consortium, Cleveland, $\mathrm{OH}$, USA opioid-related adverse events (ORAEs) that can result in worse patient outcomes. Oliceridine, a new intravenous $\mu$-opioid receptor agonist, is shown in nonclinical studies to be biased for G protein signaling (achieving analgesia) with limited recruitment of $\beta$-arrestin (associated with ORAEs). In two phase 3 randomized controlled studies of patients with moderate-to-severe acute pain following hard or soft tissue surgery, in which analgesia was measured using Sum of Pain Intensity Differences (SPID) from baseline over 48 and $24 \mathrm{~h}$ (SPID-48 and -24 respectively, oliceridine at demand doses of $0.1,0.35$, or $0.5 \mathrm{mg}$ was highly effective compared to placebo, with a favorable safety profile compared to morphine. This exploratory analysis was conducted to determine whether the safety benefits seen with oliceridine persisted when adjusted for equal levels of analgesia compared to morphine.

Methods: Presence of at least one treatmentemergent ORAE (based on Medical Dictionary for Regulatory Activities [MedDRA]-coded events: hypoxemia, nausea, vomiting, sedation, pruritus, or dizziness) was used as the composite safety endpoint. A logistic regression model was utilized to compare oliceridine (pooled regimens) versus morphine, after controlling for analgesia (using SPID-48 or SPID-24 with prerescue scores carried forward $6 \mathrm{~h}$ ). This analysis excluded patients receiving placebo and was repeated for each study and for pooled data.

Results: At a given level of SPID-48 or SPID-24, patients receiving oliceridine were less likely to 
experience the composite safety endpoint. Although not statistically significant at the 0.05 level in the soft tissue model, the odds ratio (OR) showed a consistent numerical trend for oliceridine, being approximately half that observed with morphine in both the hard (OR 0.499; 95\% confidence interval [CI] 0.255, 0.976; $p=0.042$ ) and soft (OR 0.542; 95\% CI 0.250, 1.175; $p=0.121$ ) tissue studies. Results from the pooled data were consistent with those observed in the individual studies (OR 0.507; 95\% CI 0.304, 0.844; $p=0.009$ ). Conclusion: Findings from this exploratory analysis suggest that at comparable levels of analgesia, patients receiving oliceridine were less likely to experience the composite safety endpoint consisting of ORAEs compared to patients treated with morphine.

Keywords: Adverse events; Logistic regression; Oliceridine; Opioid analgesic

\section{Key Summary Points}

\section{Why carry out this study?}

Oliceridine, a new intravenous opioid, is shown in nonclinical studies to be a $G$ protein-biased agonist at the $\mu$-opioid receptor. This preferential activity may result in potent analgesia with reduced recruitment of $\beta$-arrestin, a signaling pathway associated with opioid-related adverse events (ORAEs).

In two randomized, placebo- and activecontrolled phase 3 studies, we have previously reported that oliceridine administered via patient-controlled analgesia provided effective analgesia with a favorable safety profile.

An understanding of the relationship between efficacy and safety is important to assess the benefit-risk profile of a new compound. Randomized clinical trials are typically designed to evaluate efficacy and the statistical analytical methods used may be underpowered to identify any differences on the adverse event profile between treatments.
Logistic regression offers a framework that allows an estimate of the probability of an adverse reaction associated with a treatment, including the assessment of the strength of association. It is routinely used in the evaluation of post-marketing adverse effects reports to determine association of causality.

In this exploratory analysis, we utilized the logistic regression method to evaluate the safety of oliceridine when adjusted for equal levels of analgesia compared to morphine using data from the two pivotal randomized clinical trials. The ORAEs of nausea, vomiting, sedation, dizziness, pruritus, and hypoxia, with at least one treatment-emergent adverse event, was used as the composite safety endpoint.

\section{What was learned from the study?}

The analysis demonstrates that at comparable levels of analgesia, patients receiving oliceridine were less likely to experience the composite safety endpoint consisting of ORAEs compared to patients treated with morphine.

\section{DIGITAL FEATURES}

This article is published with digital features, including a video, to facilitate understanding of the article. To view digital features for this article go to https://doi.org/10.6084/m9. figshare.16895869.

\section{INTRODUCTION}

Nearly $86 \%$ of surgical patients report experiencing acute pain during the postoperative period, and $75 \%$ of these patients report the pain intensity as moderate to severe [1]. Inadequate control of postoperative pain can have negative consequences, including increased morbidity, impaired physical function and quality of life, slowed recovery, increased length 
of hospitalization, and increased cost of care [2]. While the use of opioids remains a key component of postoperative pain management $[3,4]$, in recent years, implementation of Enhanced Recovery After Surgery (ERAS) protocols to optimize patient outcomes have included strategies for decreasing postoperative pain via a multimodal, opioid-sparing approach $[4,5]$. However, many multimodal analgesic protocols under the ERAS guidelines have provided inconsistent evidence on postoperative opioid-sparing abilities or adequate pain control $[4,6,7]$. Recent studies also report increased adverse events (AEs) associated with the use of non-opioid strategies $[8,9]$. The US Centers for Disease Control and Prevention recommend that in acute postsurgical pain, the benefits of a limited course of opioids may outweigh the risks if pain management is inadequate with non-opioid therapies [10]. Thus, defining the rational use of opioids with a focus on the optimal balance of analgesia with minimal risks is important [11].

Conventional opioids have a narrow therapeutic index and are associated with dose-limiting opioid-related AEs (ORAEs), such as nausea, vomiting, pruritus, constipation, or respiratory depression [12-14]. Oliceridine, a new intravenous (IV) opioid, is shown in nonclinical studies to be a $G$ protein-biased agonist at the $\mu$-opioid receptor. This $G$ protein bias may result in analgesia with limited recruitment of $\beta$ arrestin, a signaling pathway associated with ORAEs [15]. In two randomized, doubleblind, placebo- and morphine-controlled studies conducted in patients with moderate-tosevere acute pain following either orthopedic surgery (hard tissue-bunionectomy) or plastic surgery (soft tissue-abdominoplasty), oliceridine administered using patient-controlled analgesia (PCA) at demand doses of 0.1, 0.35, and $0.5 \mathrm{mg}$ was highly effective compared to placebo and had a favorable safety profile $[16,17]$. Exploratory analyses indicated that the oliceridine 0.35 - and 0.5 -mg demand dose regimens were noninferior to morphine.

Measurement of respiratory safety burden (RSB) calculated as the mathematical product of the incidence of a defined set of observed respiratory safety events (RSE) multiplied by the mean expected cumulative duration of these events (in hours) was a key secondary endpoint in both studies $[16,17]$. In both studies, the incidences of the RSE were numerically lower for each of the oliceridine dosage regimens compared to the morphine regimen, although statistical significance was not reached. Also, the overall rate of the RSEs was lower than expected in all the treatment groups (oliceridine, morphine, and placebo regimens), possibly resulting in a reduced statistical power to detect any significant differences between treatment $[16,17]$. In both studies, spontaneously reported AEs were assessed during the randomized treatment and 7-day follow-up period, and coded using the Medical Dictionary for Regulatory Activities (MedDRA), version $19.0[16,17]$. In the bunionectomy trial (hardtissue model), the overall reported incidence of AEs ranged from 73.7 to $91.1 \%$ in the oliceridine treatment regimens and was $96.1 \%$ in the morphine group and $68.4 \%$ in the placebo group. Similar findings were observed in the abdominoplasty study (soft-tissue model), with the reported incidence of overall AEs ranging from 89.6 to $95.0 \%$ in the oliceridine treatment regimens, and $97.6 \%$ in the morphine group and $78.3 \%$ in the placebo group. In both studies, the most common treatment-emergent AEs (reported in $\geq 10 \%$ of patients) in any treatment group were nausea, vomiting, dizziness, headache, constipation, pruritus, and hypoxia.

An understanding of the relationship between efficacy and safety is important to assess the benefit-risk profile. Typically, in a randomized clinical trial (RCT), the statistical analytical methods to evaluate efficacy are well established but this is not the case for adverse drug reactions [18]. One of the challenges in applying statistical analysis to AEs is that RCTs are typically designed to evaluate efficacy and the statistical analytical methods used are underpowered to detect differences in AEs between treatments-unless it is a "predefined" measure $[18,19]$. In any RCT, spontaneously reported AEs are typically collected and reported as descriptive measures. The descriptive summaries provide information on the "detecting signals" of AEs [19] but do not allow comparison between treatments. 
Logistic regression offers a framework that allows an estimate of the probability of an adverse reaction associated with a treatment, including the assessment of the strength of association, and is routinely used in the evaluation of post-marketing $\mathrm{AE}$ reports to determine association of causality $[20,21]$. Based on findings from a phase 1 study and a fixed-dose phase 2 study, an initial $1 \mathrm{mg}$ dose of oliceridine is approximately equipotent to morphine $5 \mathrm{mg}$ $[22,23]$. However, the dose equivalency of oliceridine to morphine after cumulative dosing has not been established. Thus, in this analysis, we utilized the logistic regression method to evaluate the safety of oliceridine when adjusted for equal levels of analgesia (as measured by Sum of Pain Intensity Differences [SPID]) compared to morphine using data from the two pivotal RCTs, APOLLO-1 (orthopedic surgerybunionectomy) [17] and APOLLO-2 (plastic surgery-abdominoplasty) [16]. The findings from this exploratory analysis are reported here.

\section{METHODS}

The study design and the results of the randomized, double-blind, placebo- and morphinecontrolled studies following either orthopedic surgery (hard-tissue model-bunionectomy) or plastic surgery (soft-tissue model-abdominoplasty) were previously described $[16,17]$ (See Electronic Supplementary Material Fig. 1: Study designs of the phase 3 pivotal trials). Both studies were conducted in compliance with the protocol and regulatory requirements consistent with the International Council on Harmonization Good Clinical Practice Guidelines and the ethical principles of the Declaration of Helsinki. The study was approved by an Institutional Review Board at each investigational site, and patients provided written informed consent before participation. Advarra Inc. (Columbia, MD, USA) provided a centralized institutional review board approval for all sites.

Although similar in design, these two studies differed in their use of anesthetics (regional anesthesia with popliteal sciatic nerve block in the bunionectomy study and general anesthesia with the use of fentanyl and propofol in the abdominoplasty study), time from surgery to the first dose of study medication, temporal courses of pain after discontinuation of anesthesia (within $9 \mathrm{~h}$ after discontinuation of regional anesthesia in the bunionectomy study, and within $4 \mathrm{~h}$ after surgery and at least $20 \mathrm{~min}$ after the last dose of fentanyl in the abdominoplasty study), qualifying numerical rating scale (NRS) pain intensity scores, and duration of the randomized treatment period ( $48 \mathrm{~h}$ for the bunionectomy study and $24 \mathrm{~h}$ for the abdominoplasty study).

In both studies, patients aged 18-75 years, with a body mass index $\leq 35 \mathrm{~kg} / \mathrm{m}^{2}$ and body weight $\geq 40 \mathrm{~kg}$, who underwent primary surgery of either bunionectomy (orthopedic surgery) or abdominoplasty (plastic surgery) and experienced moderate-to-severe pain as measured by the NRS ( $\geq 4$ for the bunionectomy study and $\geq 5$ for the abdominoplasty study) were enrolled. Patients were randomized to receive IV demand dose regimens of either placebo, oliceridine $0.1 \mathrm{mg}$, oliceridine $0.35 \mathrm{mg}$, oliceridine $0.5 \mathrm{mg}$, or morphine $1 \mathrm{mg}$. For each regimen, a clinician administered a fixed IV loading dose (oliceridine $1.5 \mathrm{mg}$, morphine $4 \mathrm{mg}$, or volume-matched placebo) followed by demand doses administered via a PCA device and blinded supplemental doses. PCA doses were allowed from $10 \mathrm{~min}$ after the loading dose and limited by a 6-min lockout interval. Blinded clinician-administered IV supplemental doses (oliceridine $0.75 \mathrm{mg}$ and morphine $2 \mathrm{mg}$ ) were permitted as often as hourly as needed (PRN). The dosing limit for all groups was three PCA syringes or six clinician-administered supplemental PRN doses within the first $12 \mathrm{~h}$ (60 $\mathrm{mg}$ for the oliceridine group). Patients reaching this dosing limit were discontinued, managed with conventional therapies, and allowed to remain in the study as nonresponders.

In both studies, RSB experienced by patients in each treatment group, calculated as the mathematical product of the incidence of a defined set of observed RSEs multiplied by the mean expected cumulative duration of these events (RSB; in hours), was a prespecified endpoint and findings have been previously reported $[16,17]$. 
Both studies also reported overall safety and tolerability assessed via the occurrence of treatment-emergent AEs that were spontaneously reported during the randomized treatment and 7-day follow-up period, coded using MedDRA, version 19.0. For this exploratory analysis, the spontaneously reported MedDRA events, in particular, events that occurred in $\geq 10 \%$ of patients in any treatment group of oliceridine or morphine, was utilized. Patients receiving placebo were excluded from the analysis. The ORAEs (MedDRA-coded events) of nausea, vomiting, sedation, dizziness, pruritus, and hypoxia, with at least one treatment-emergent $\mathrm{AE}$, was used as the composite safety endpoint. The AEs of oliceridine and morphine, adjusted for therapeutic effectiveness (analgesia as measured by SPID is held constant across treatment groups) were compared by logistic regression.

\section{Statistical Methodology}

In the logistic regression model, the presence of at least one treatment-emergent $\mathrm{AE}$ as mentioned above (the composite safety endpoint) was defined as "Yes"; and absence of the AE was designated as "No." Analgesia was determined utilizing the SPID-48 (SPID from baseline over $48 \mathrm{~h}$ ) for the bunionectomy (hard-tissue surgery) and SPID-24 (SPID from baseline over 24 h) for the abdominoplasty (soft-tissue surgery) studies. For patients who received rescue analgesics (etodolac $200 \mathrm{mg}$ every $6 \mathrm{~h}$ [q6h] as needed), the pre-rescue pain score was used instead of the pain scores measured after rescue medication usage for $6 \mathrm{~h}$. This imputation was utilized in the calculation of the SPID score.

The logistic regression model included the effects of treatment, baseline pain score, and SPID-48/SPID-24. This analysis was done for both individual studies as well as on the pooled data. Interaction terms were included to explore the consistency of the effect across treatment groups. For the individual studies, the composite safety endpoint was the dependent variable, with treatment, baseline pain score, SPID-48 or SPID-24, and the interaction terms of treatment by baseline pain score, treatment by SPID-48/ SPID-24, and baseline pain score by SPID-48/
SPID-24 as explanatory variables. For the pooled data, the logistic regression model included the same dependent variable (composite safety endpoint) and all of the explanatory variables as for the individual studies, with the addition of study indicator interactions into the model. Additional interaction terms utilizing the study indicator were added to explore the consistency of the effect across the two studies. For ease of interpretation, treatment groups were dichotomized since patients could achieve the same amount of oliceridine despite the demand dose regimen. In the model, oliceridine demand dose regimens of $0.1,0.35$, and $0.5 \mathrm{mg}$ were pooled and set as "zero" and morphine treatment regimen was set to "one." A backward elimination methodology was utilized across all analyses using the $p \leq 0.15$ criteria for determining the final model.

For the individual studies, we also evaluated the effects of treatment when adjusted for equal levels of analgesia on the AEs of nausea, vomiting, sedation, dizziness, pruritus, and hypoxia.

\section{RESULTS}

The incidence of spontaneously reported MedDRA events (nausea, vomiting, sedation, dizziness, pruritus, and hypoxia) used in the composite endpoint in any of the oliceridine or morphine treatment groups by study is shown in Table 1.

\section{Composite Safety Endpoint}

The odds ratio (OR) for the logistic regression model for individual studies and pooled data is shown in Table 2. The OR with its associated 95\% confidence interval (CI) for the effect of treatment were 0.499 (95\% CI $0.255,0.976)$ and 0.542 (95\% CI $0.250,1.175)$ in the bunionectomy and abdominoplasty studies, respectively (Fig. 1). In the abdominoplasty study, the effect of baseline pain score on the composite safety endpoint was also statistically significant at the 0.05 alpha level (OR 1.279; 95\% CI 1.037, 1.576). The findings from the individual studies suggest that at any given level of SPID- 48 or SPID-24, the OR for the oliceridine composite 
Table 1 Incidence of spontaneously reported adverse events, coded using the Medical Dictionary for Regulatory Activities version 19.0, used in the composite safety endpoint

\begin{tabular}{|c|c|c|c|c|}
\hline \multicolumn{5}{|c|}{ Orthopedic (hard-tissue) surgery_bunionectomy (APOLLO-1) [17] } \\
\hline \multirow[t]{2}{*}{ Adverse drug reaction, $n(\%)$} & \multicolumn{3}{|c|}{ Oliceridine demand dose regimen } & \multirow[t]{2}{*}{ Morphine $1 \mathrm{mg}(n=76)$} \\
\hline & $0.1 \mathrm{mg}(\mathrm{n}=76)$ & $0.35 \mathrm{mg}(n=79)$ & $0.5 \mathrm{mg}(n=79)$ & \\
\hline Nausea & $27(35.5)$ & $44(55.7)$ & $50(63.3)$ & $49(64.5)$ \\
\hline Vomiting & $13(17.1)$ & $31(39.2)$ & $32(40.5)$ & $38(50.0)$ \\
\hline Sedation & $6(7.9)$ & $19(24.1)$ & $12(15.2)$ & $12(15.8)$ \\
\hline Dizziness & $21(27.6)$ & $25(31.6)$ & $28(35.4)$ & $26(34.2)$ \\
\hline Pruritus & $2(2.6)$ & $13(16.5)$ & $5(6.3)$ & $24(31.6)$ \\
\hline Hypoxia & 0 & $4(5.1)$ & $7(8.9)$ & $7(9.2)$ \\
\hline
\end{tabular}

Plastic (soft-tissue) surgery-abdominoplasty (APOLLO-2) [16]

\begin{tabular}{lllll}
\hline Adverse drug reaction, $\boldsymbol{n}(\%)$ & \multicolumn{2}{c}{ Oliceridine demand dose regimen } & Morphine 1 mg $(\boldsymbol{n}=\mathbf{8 2})$ \\
\cline { 2 - 4 } & $\mathbf{0 . 1} \mathbf{~} \mathbf{~ g ~}(\boldsymbol{n}=\mathbf{7 7})$ & $\mathbf{0 . 3 5} \mathbf{~} \mathbf{g}(\boldsymbol{n}=\mathbf{7 9 )}$ & $\mathbf{0 . 5} \mathbf{~ m g ~}(\boldsymbol{n}=\mathbf{8 0})$ & \\
\hline Nausea & $34(44.2)$ & $49(62.0)$ & $60(75.0)$ & $61(74.4)$ \\
Vomiting & $18(23.4)$ & $17(21.5)$ & $34(42.5)$ & $44(53.7)$ \\
Sedation & $7(9.1)$ & $11(13.9)$ & $11(13.8)$ & $25(30.5)$ \\
Dizziness & $11(14.3)$ & $7(8.9)$ & $7(8.8)$ & $13(15.9)$ \\
Pruritus & $11(14.3)$ & $14(17.7)$ & $14(17.5)$ & $19(23.2)$ \\
Hypoxia & $6(7.8)$ & $16(20.3)$ & $14(17.5)$ & $19(23.2)$
\end{tabular}

Table is adapted from $[16,17]$

Adverse events were spontaneously reported with onset at the time of or following the initiation of the loading dose with study medication until 7 days after the last dose of study medication. The placebo treatment arm is not shown in the table as patients receiving placebo were excluded from the analysis

safety endpoint was approximately half of that observed with morphine (Table 2; Fig. 1). In the pooled data, the OR estimate was 0.507 (95\% CI $0.304,0.844)$ for the treatment effect (Table 2; Fig. 1).

\section{Individual ORAEs}

The influence of treatment effect on the individual ORAEs of nausea, vomiting, sedation, dizziness, pruritus, and hypoxia for the individual studies are shown in Fig. 2. In the bunionectomy study, the OR was lower (OR < 1) for five of six individual AEs, with favorability towards oliceridine over morphine for nausea, vomiting, and pruritus; the ORs (95\%
CIs) for these latter three AEs were $0.544(0.308$, $0.964), 0.464(0.268,0.804)$, and 0.319 (0.150, 0.674 ) (Fig. 2). In the abdominoplasty study, the ORs were lower for all of the six individual AEs with oliceridine versus morphine, with favorability towards oliceridine for nausea (OR 0.526 ; 95\% CI 0.289, 0.959), vomiting (OR $0.351 ; 95 \%$ CI $0.208,0.595$ ), and sedation (OR 0.385; 95\% CI 0.195, 0.762) (Fig. 2).

\section{DISCUSSION}

Oliceridine is structurally distinct from natural opiates (e.g., morphine) and from its semi-synthetic derivatives (e.g., hydromorphone) [24]. 
Table 2 Final logistic regression model for individual studies and pooled data

\begin{tabular}{llll}
\hline Effect & Odds ratio estimate & 95\% Confidence interval (lower, upper) & $\boldsymbol{p}$ value \\
\hline $\begin{array}{l}\text { Orthopedic (hard tissue) surgery-bunionectomy study } \\
\text { Treatment (oliceridine vs. morphine) }\end{array}$ & 0.499 & $0.255,0.976$ & 0.0422 \\
$\begin{array}{l}\text { Plastic (soft tissue) surgery_abdominoplasty study } \\
\text { Treatment (oliceridine vs. morphine) }\end{array}$ & 0.542 & $0.250,1.175$ & \\
Baseline pain score & 1.279 & $1.037,1.576$ & 0.1209 \\
Analysis from the pooled studies & & & 0.0213 \\
Treatment (oliceridine vs. morphine) & 0.507 & $0.304,0.844$ & 0.009 \\
\hline
\end{tabular}

a The final model included treatment, baseline pain score, and Sum of Pain Intensity Differences (SPID) from baseline to $48 \mathrm{~h}$ (SPID-48) as main effects, and the baseline pain score and SPID-48 interaction

b The final model included treatment and baseline pain score as main effects

c The final model included treatment, study identifier, baseline pain score, and SPID-48 or SPID from baseline to $24 \mathrm{~h}$ (SPID-24), baseline pain score and study identifier interaction, and baseline pain score and SPID-48/-24 interaction

Odds Ratio with $95 \% \mathrm{Cl}$

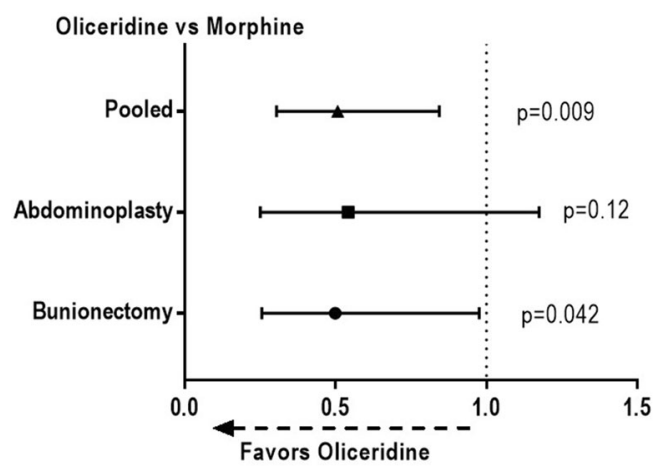

Fig. 1 Odds ratio and $95 \%$ confidence interval $(C I)$ for treatment effect (oliceridine vs. morphine) on the composite endpoint. The composite safety endpoint was defined as the presence of at least one treatment-emergent adverse event of nausea, vomiting, sedation, dizziness, pruritus, or hypoxia, as coded by the Medical Dictionary for Regulatory Activities version 19.0. In the model, oliceridine demand dose regimens of $0.1,0.35$, and $0.5 \mathrm{mg}$ were pooled and set as "zero," and the morphine treatment regimen was set to "one." The duration was $48 \mathrm{~h}$ for the bunionectomy study and $24 \mathrm{~h}$ for the abdominoplasty study. Analgesia as measured by the Sum of Pain Intensity Difference (SPID) is held constant across treatment groups; SPID-48 (SPID from baseline to $48 \mathrm{~h}$ ) and SPID-24 (SPID from baseline to $24 \mathrm{~h}$ ) were used for the bunionectomy and abdominoplasty studies, respectively
The development of oliceridine stemmed from the concept that a $G$ protein-biased ligand with reduced $\beta$-arrestin recruitment might offer increased analgesia with improved safety and tolerability [25]. Although preclinical findings support the hypothesis of "biased agonist" $[15,26]$, the translation of these findings in humans has not been fully established. In a clinical setting, when the aim is to manage moderate-to-severe acute pain, what is important is the availability of good analgesic efficacy with a favorable side effect profile [27]. Using the logistic regression model, the findings from the individual studies suggest that at any given level of SPID-48 or SPID-24, the OR for oliceridine on the composite safety endpoint was approximately half of that observed with morphine. The results from the analysis of the pooled data were consistent with the findings from individual studies and show that, at any given SPID-48/-24, patients receiving oliceridine were less likely to experience the defined safety composite endpoint. Findings from this exploratory analysis suggest that under equianalgesic conditions, oliceridine has a favorable risk-benefit profile when compared to morphine.

In both of the pivotal RCTs $[16,17]$, findings from exploratory analyses indicated that the 


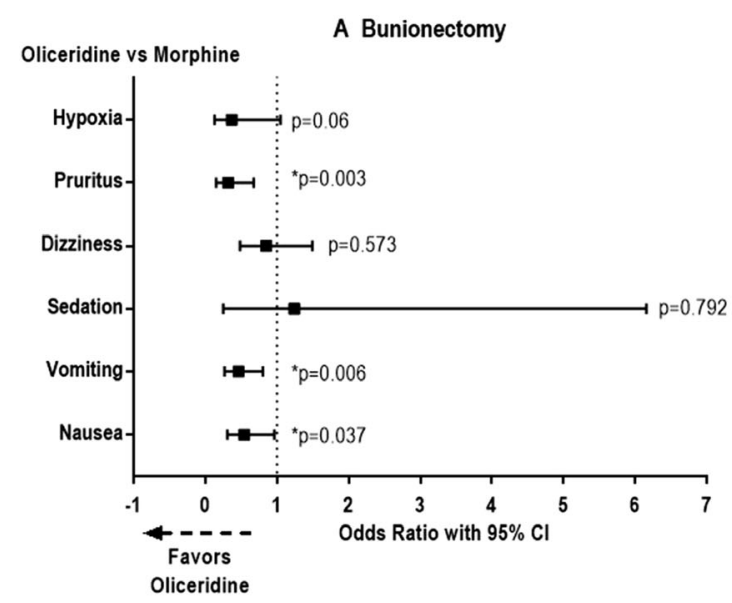

Fig. 2 Odds ratio and 95\% CI for treatment effect (oliceridine vs. morphine) onindividual adverse events. In the model, oliceridine demand dose regimens of $0.1,0.35$, and $0.5 \mathrm{mg}$ were pooled and set as "zero," and morphine treatment regimen was set to "one." The duration was $48 \mathrm{~h}$

oliceridine $0.35 \mathrm{mg}$ and $0.5 \mathrm{mg}$ demand doses were noninferior to the morphine $1 \mathrm{mg}$ demand dose. The most common AEs reported in both these trials were mild to moderate in intensity, and the incidence of AEs leading to early study medication discontinuation was low [16, 17]. The composite safety endpoint included in this analysis was the most common AEs reported in $\geq 10 \%$ of patients and included nausea, vomiting, sedation, dizziness, pruritus, and hypoxia. Among these events, the reported incidence of hypoxia was $<10 \%$ in the bunionectomy study, with rates reported in 5\% of patients with the oliceridine $0.35 \mathrm{mg}$ demand dose regimen and in $9 \%$ of patients with the oliceridine $0.5 \mathrm{mg}$ and morphine $1 \mathrm{mg}$ demand dose regimens, respectively. Thus, in the bunionectomy study the contribution of hypoxia to the overall composite safety endpoint appears to be minimal. The incidence of sedation/somnolence, although similar for oliceridine in both phase 3 randomized controlled studies, was higher for morphine in the abdominoplasty study than in the bunionectomy study. In the bunionectomy study the incidence of sedation/somnolence for morphine was comparable to that for the oliceridine treatment and, consequently, the logistic regression model did not show a treatment

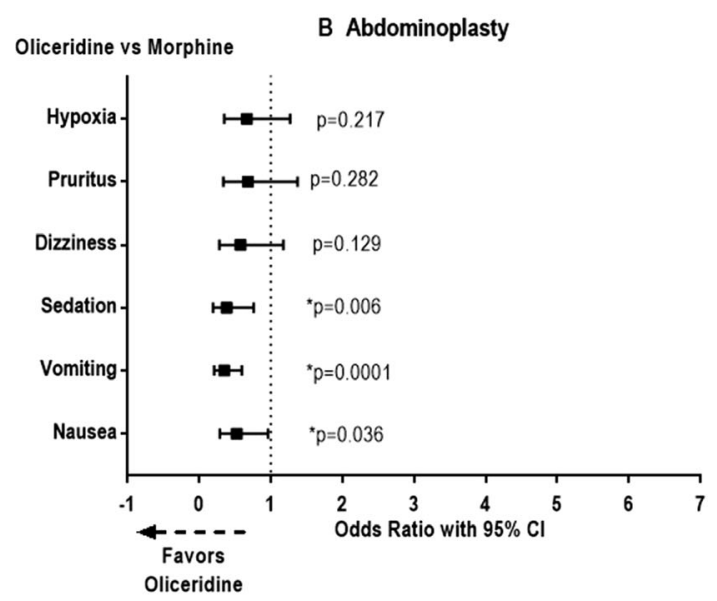

for the bunionectomy study and $24 \mathrm{~h}$ for the abdominoplasty study. Analgesia as measured by SPID was held constant across treatment groups; SPID-48 and SPID-24 were used for the bunionectomy and abdominoplasty studies, respectively

difference; in contrast, in the abdominoplasty study a treatment effect in favor of oliceridine was observed.

Analysis of the individual AEs did show that nausea and vomiting were the most common AEs in both studies, contributing to the significant treatment difference between oliceridine and morphine. In another earlier exploratory analysis on an endpoint of complete gastrointestinal (GI) response defined as "no vomiting and no use of rescue medication," we reported that when controlled for the analgesic effects (constant SPID-48/-24), the odds of achieving a complete GI response was higher with oliceridine than with morphine, suggesting better GI tolerability with oliceridine [28]. The findings from this analysis further confirm a favorable benefit-risk profile for oliceridine as it relates to the AEs of nausea and vomiting. Of note, in both of these randomized controlled clinical studies [16, 17], the majority of the enrolled patients had an Apfel score $\geq 3$ (with similar distribution across treatment arms), indicating a high risk for postoperative nausea and vomiting (PONV). The guidelines for the management of PONV also recommend use of three to four prophylactic antiemetics in patients with $>2$ risk factors. Both of the randomized controlled clinical trials did not permit the use of any 
prophylactic antiemetics. Thus, the findings of a favorable risk profile with oliceridine is compelling.

There are certain limitations to our analysis. The analysis is exploratory in nature and the findings are post-randomization. The AEs collected were spontaneously reported, and a formal assessment of the AEs included in the composite safety endpoint was not conducted. Incorporating objective statistical methods helps to assist the evaluation of AE information to better identify benefit-risk relationships [18].

\section{CONCLUSIONS}

The findings from this exploratory analysis suggest that when analgesia (as measured by SPID-48 and SPID-24) is held constant across treatment groups, patients receiving oliceridine were less likely to experience AEs compared to patients treated with morphine. In both studies, the OR for the composite safety endpoint with oliceridine was approximately half of that observed with morphine. Findings from the analysis show that under equianalgesic conditions, oliceridine has a favorable risk-benefit profile compared to morphine.

\section{ACKNOWLEDGEMENTS}

The authors wish to thank Kanaka Sridharan, M.S., R.Ph., an employee at Trevena Inc., for editorial assistance and creation of the figures.

Funding. The studies included here in the exploratory analyses (orthopedic surgerybunionectomy, APOLLO-1; ClinicalTrials.gov Identifier: NCT02815709) and plastic surgeryabdominoplasty, APOLLO-2; ClinicalTrials.gov Identifier: NCT02820324) and the journal's Rapid Service Fee were sponsored by Trevena, Inc., Chesterbrook, PA, USA. The exploratory analysis described here was also funded by Trevena, Inc.

Authorship. All named authors meet the International Committee of Medical Journal Editors (ICMJE) criteria for authorship for this article, take responsibility for the integrity of the work as a whole, and have given their approval for this version to be published.

Authorship Contributions. Mark A Demitrack and Michael J Fossler provided the concept and design for the analysis, and interpreted the results. Gregory Hammer, Sabry Ayad, Ashish Khanna, and Linda Wase provided inputs to the design of the analysis and interpretation of the results. Roderick Little and Cathy Michalsky performed the statistical analysis. Gregory Hammer, Sabry Ayad, and Ashish Khanna provided direction for the Introduction and Discussion section of the manuscript; Cathy Michalsky drafted the Methods and Results sections. Gregory Hammer, Sabry Ayad, Ashish Khanna, Roderick Little, Mark A Demitrack, Michael J Fossler, and Linda Wase revised the draft critically for scientific content.

Disclosures. Dr. Gregory Hammer and Dr. Sabry Ayad serve in the Speakers' Bureau. Dr. Roderick Little served as a consultant for Trevena and provided direction to the conduct of the analysis. Dr. Ashish Khanna is a consultant to Trevena, Inc. Linda Wase, Cathy Michalsky, Mark A. Demitrack, and Michael J. Fossler, are full-time employees and stockholders of Trevena Inc.

Compliance with Ethics Guidelines. All procedures performed in the studies were conducted in accordance with the International Conference on Harmonization Good Clinical Practice guidelines and ethical principles that have their origin in the Declaration of Helsinki. The study was approved by an Institutional Review Board at each investigational site, and patients provided written informed consent before participation. Advarra ${ }^{\circledR}$ provided a centralized institutional review board approval for all sites. Informed consent was obtained from all individual participants included in the study, as specified in the APOLLO-1 and APOLLO-2 studies.

Data Availability. The data sets generated during and/or analyzed during the current 
study are available from Trevena, Inc. on reasonable request.

Open Access. This article is licensed under a Creative Commons Attribution-NonCommercial 4.0 International License, which permits any non-commercial use, sharing, adaptation, distribution and reproduction in any medium or format, as long as you give appropriate credit to the original author(s) and the source, provide a link to the Creative Commons licence, and indicate if changes were made. The images or other third party material in this article are included in the article's Creative Commons licence, unless indicated otherwise in a credit line to the material. If material is not included in the article's Creative Commons licence and your intended use is not permitted by statutory regulation or exceeds the permitted use, you will need to obtain permission directly from the copyright holder. To view a copy of this licence, visit http://creativecommons.org/licenses/by$\mathrm{nc} / 4.0 /$.

\section{REFERENCES}

1. Gan TJ, Habib AS, Miller TE, White W, Apfelbaum JL. Incidence, patient satisfaction, and perceptions of post-surgical pain: results from a US national survey. Curr Med Res Opin. 2014;30:149-60.

2. Gan TJ. Poorly controlled postoperative pain: prevalence, consequences, and prevention. J Pain Res. 2017;10:2287-98.

3. Small C, Laycock H. Acute postoperative pain management. Br J Surg. 2020;107:e70-80.

4. Echeverria-Villalobos M, Stoicea N, Todeschini AB, et al. Enhanced Recovery After Surgery (ERAS): a perspective review of postoperative pain management under ERAS pathways and its role on opioid crisis in the United States. Clin J Pain. 2020;36: 219-26.

5. Chakravarthy V, Yokoi H, Manlapaz MR, Krishnaney AA. Enhanced recovery in spine surgery and perioperative pain management. Neurosurg Clin $\mathrm{N}$ Am. 2000;31:81-91.

6. Bernstein J, Feng J, Mahure S, Schwarzkopf R, Long W. Revision total knee arthroplasty is associated with significantly higher opioid consumption as compared with primary total knee arthroplasty in the acute postoperative period. Arthroplast Today. 2020;6:172-5.

7. Maheshwari K, Avitsian R, Sessler DI, et al. Multimodal analgesic regimen for spine surgery: a randomized placebo-controlled trial. Anesthesiology. 2020;132:992-1002.

8. Beloeil H, Garot M, Lebuffe G, et al. Balanced opioid-free anesthesia with dexmedetomidine versus balanced anesthesia with remifentanil for major or intermediate noncardiac surgery. Anesthesiology. 2021;134:541-51.

9. Verret M, Lauzier F, Zarychanski R, et al. Perioperative use of gabapentinoids for the management of postoperative acute pain: a systematic review and meta-analysis. Anesthesiology. 2020;133:265-79.

10. US Centers for Disease Control and Prevention (CDC). Postsurgical pain. 2020. https://www. cdc.gov/acute-pain/postsurgical-pain/index.html. Accessed 24 May 2021.

11. Kharasch ED, Clark JD. Opioid-free anesthesia: time to regain our balance. Anesthesiology. 2021;134: 509-14.

12. Shafi S, Collinsworth AW, Copeland LA, et al. Association of opioid-related adverse drug events with clinical and cost outcomes among surgical patients in a large integrated health care delivery system. JAMA Surg. 2018;153:757-63.

13. Oderda GM, Senagore AJ, Morland K, et al. Opioidrelated respiratory and gastrointestinal adverse events in patients with acute postoperative pain: prevalence, predictors, and burden. J Pain Palliat Care Pharmacother. 2019;33:82-97.

14. Overholser BR, Foster DR. Opioid pharmacokinetic drug-drug interactions. Am J Manag Care. 2011;17(Suppl 11):S276-87.

15. DeWire SM, Yamashita DS, Rominger DH, et al. A G protein-biased ligand at the mu-opioid receptor is potently analgesic with reduced gastrointestinal and respiratory dysfunction compared with morphine. J Pharmacol Exp Ther. 2013;344:708-17.

16. Singla NK, Skobieranda F, Soergel DG, et al. APOLLO-2: a randomized, placebo and active-controlled phase iii study investigating oliceridine (TRV130), a G protein-biased ligand at the muopioid receptor, for management of moderate to severe acute pain following abdominoplasty. Pain Pract. 2019;19:715-31.

17. Viscusi ER, Skobieranda F, Soergel DG, Cook E, Burt DA, et al. APOLLO-1: a randomized placebo and active-controlled phase III study investigating 
oliceridine (TRV130), a G protein-biased ligand at the micro-opioid receptor, for management of moderate-to-severe acute pain following bunionectomy. J Pain Res. 2019;12:927-43.

18. Phillips R, Hazell L, Sauzet O, Cornelius V. Analysis and reporting of adverse events in randomised controlled trials: a review. BMJ Open. 2019;9: e024537.

19. Phillips R, Sauzet O, Cornelius V. Statistical methods for the analysis of adverse event data in randomised controlled trials: a scoping review and taxonomy. BMC Med Res Methodol. 2020;20:288.

20. Van Holle L, Bauchau V. Use of logistic regression to combine two causality criteria for signal detection in vaccine spontaneous report data. Drug Saf. 2014;37:1047-57.

21. Duan JZ. Two commonly used methods for exposure-adverse events analysis: comparisons and evaluations. J Clin Pharmacol. 2009;49:540-52.

22. Soergel DG, Subach RA, Burnham N, et al. Biased agonism of the mu-opioid receptor by TRV130 increases analgesia and reduces on-target adverse effects versus morphine: a randomized, doubleblind, placebo-controlled, crossover study in healthy volunteers. Pain. 2014;155:1829-35.
23. Viscusi ER, Webster L, Kuss M, et al. A randomized, phase 2 study investigating TRV130, a biased ligand of the mu-opioid receptor, for the intravenous treatment of acute pain. Pain. 2016;157:264-72.

24. Gan TJ, Wase L. Oliceridine, a G protein-selective ligand at the $\mu$-opioid receptor, for the management of moderate to severe acute pain. Drugs Today (Barc). 2020;56:269-86.

25. Violin JD, Crombie AL, Soergel DG, Lark MW. Biased ligands at G-protein-coupled receptors: promise and progress. Trends Pharmacol Sci. 2014;35: 308-16.

26. Liang DY, Li WW, Nwaneshiudu C, Irvine KA, Clark JD. Pharmacological characters of oliceridine, a muopioid receptor G-protein-biased ligand in mice. Anesth Analg. 2019;129:1414-21.

27. Lambert D, Calo G. Approval of oliceridine (TRV130) for intravenous use in moderate to severe pain in adults. Br J Anaesth. 2000;125:e473-4.

28. Beard TL, Michalsky C, Candiotti KA, et al. Oliceridine is associated with reduced risk of vomiting and need for rescue antiemetics compared to morphine: exploratory analysis from two phase 3 randomized placebo and active controlled trials. Pain Ther. 2021;10:401-13. 